\title{
Testimony and children's acquisition of number concepts
}

\author{
Helen De Cruz
}

This is the final, non-copyedited draft of a paper now published in S. Bangu (Ed). Naturalizing Logico-Mathematical Knowledge. Approaches from Philosophy, Psychology and Cognitive Science. London: Routledge.

\begin{abstract}
An enduring puzzle in philosophy and developmental psychology is how young children acquire number concepts, in particular the concept of natural number. Most solutions to this problem conceptualize young learners as lone mathematicians who individually reconstruct the successor function and other sophisticated mathematical ideas. In this paper, I argue for a crucial role of testimony in children's acquisition of number concepts, both in the transfer of propositional knowledge (e.g., the cardinality concept), and in knowledge-how (e.g., the counting routine).
\end{abstract}

\section{The puzzle of number acquisition}

At around three-and-a-half to four years of age, children in western and other numerate cultures experience a profound shift in their understanding of numbers: they come to understand how counting works. They can use number words to denote the cardinality of collections of items in a precise fashion, by placing each item to be counted into a one-to-one correspondence with elements of a counting list, and using the last item to denote the cardinality of the set (see e.g., Sarnecka in press, Le Corre 2014). Children's acquisition of number concepts is often conceptualized in terms of individual discovery and personal reconstruction. For example, Carey $(2009,302)$ writes that children learn to individuate three items "before figuring out how the numeral list represents natural number". Davidson et al. $(2012,163)$ put it this way, "sometime between the ages of 3 -and-a-half and 4, children discover that counting can be used to generate sets of the correct size for any word in their count list" (emphasis added in both).

While young children's understanding of natural numbers is remarkable, it is misleading to suggest that each child figures this out individually. Young children grow up in an environment with numerate adults and older children, who use counting systems that are transmitted over many generations. Across cultures, humans use a variety of ways to count, including tallying, body-part counting, counting rods, and abacuses. Children are born in these rich cultural environments and adopt the counting systems of their parents, further extending them and building on them. Our counting systems are in line with other cultural inventions, where successive generations build on what earlier generations have already achieved. Tomasello et al. $(2005,688)$ describe this process of learning as the ratchet effect: children are born in an environment of collective artifacts (e.g., abacuses) and social practices (e.g., body-part counting), which structure their cognitive ontogenies, and allow them to build on the cultural achievements of previous generations. It is not clear when the earliest counting systems arose, but archaeological evidence 
suggests that numeracy arose substantially earlier than writing. Bone, antlers, or ochre sticks with regular incisions appear in the archaeological record from about 77,000 years ago, but it is unclear whether these notches are purely decorative or might have numerical meaning (Cain 2006). From about 25,000 years ago, artifacts appear that have groupings of markings, indicating that quantities may have been meaningful. Two bones from Ishango, Congo (25,000 years BP) have notches that are grouped in interesting ways. For instance, one of these bones has two of its three sides covered with notches that add up to 60 (Pletser and Huylebrouck 1990).

In this paper, I will argue that testimonial transmission plays a crucial role in how children learn about numbers. This involves both transmission of propositional knowledge (knowledge-that), and the transmission of skills (knowledge-how). The paper is structured as follows: In section 2, I review children's acquisition of the cardinal principle (CP). Section 3 describes how this cognitive change is usually framed in terms of individual discovery. This view-which I term the child as a lone mathematician-fits in a broader picture of children as individual learners and discoverers. Section 4 presents an alternative account of children's learning, the child as a social learner who acquires knowledge through testimony. While traditionally (particularly in philosophy), testimonial transmission has been framed in terms of propositional knowledge, I argue for a broader view where testimonial transmission also involves demonstration and transmission of knowledge-how. In section 5 , I outline how this approach is fruitful in explaining how children acquire number concepts.

\section{Children's acquisition of the cardinal principle}

Children typically learn to count through a stable developmental sequence. Toddlers between 18 months and two years can recite counting lists (e.g., "one, two, three") as a meaningless string of words; they cannot use the number words to determine the cardinality of collections. At around two years of age, they become subsetknowers (Wynn 1990). The first stage is to be a one-knower: a child can correctly give one item when requested, e.g., "Can you give me one toy", but performs at chance level for higher quantities (e.g., the child gives three toys when two are requested). The next stage is to become a two-knower: children can correctly give one or two toys but perform at chance level for three or more; they then become three-knowers, and occasionally, four-knowers-always in that order.

One would expect that the next step is becoming a four- or five-knower, but after three or four, a crucial cognitive change takes place. Children become cardinal principle ( $\mathrm{CP}$ ) knowers: they understand that counting can be used to denote the correct cardinality of any given collection of items. The cardinal principle states that if $n$ is followed by $m$ in the counting sequence, a set with cardinality $n$ will have cardinality $m$ if an item is added. While this seems straightforward (indeed, we use this principle without conscious reflection when we count), no other animal seems to understand it, in spite of extensive efforts by experimenters to train animals in numeracy. For example, Ai, a female chimpanzee, learned to use Arabic digits 1 to 9 to denote quantities, but had to learn each separate number by brute association. She did not make the shift to CP at the numbers 4 or 5 (Biro and Matsuzawa 2001). 
CP builds on phylogenetically older numerical abilities, which we share with many other animals. Even insects, such as honeybees, can recognize small quantities up to 3 or 4 precisely, a capacity that is termed 'subitizing' (e.g., Dacke and Srinivasan 2008). Humans also subitize: they are more confident, less error-prone, and much quicker when handling small sets up to four items, compared to larger sets (Revkin et al. 2008). Human newborns can distinguish between sets up to 3 or 4 items (Antell and Keating 1983). Numerical cognition for larger quantities is approximate: for instance, we can see that putting $16+16$ together is smaller than 40 , but without counting, we don't know how much smaller (Pica et al. 2004). Young chicks (Rugani et al. 2013) and guppies (Bisazza et al. 2010) can estimate and compare different quantities, such as four versus eight without any prior training. However, animals are limited in their capacity to distinguish between numerosities larger than 3 or 4 exactly. A wide range of studies indicates that animals can distinguish between 2 and 3, but fail to tell the difference between 5 and 6 (e.g., Petrazzini et al. 2015). Animal numerical cognition above 3 or 4 is approximate, and becomes increasingly less precise as cardinalities increase (see e.g., Scarf et al. 2011, for a comparative study with pigeons and primates).

Since only humans are able to represent cardinalities above 3 or 4 precisely, cognitive scientists and philosophers have speculated how children acquire number concepts. Experimental studies on children's numerical cognition suggest that it is a mixture of knowing-that (propositional knowledge) and knowing-how (procedural knowledge). Examples of propositional knowledge are knowing that numbers can be extended indefinitely, or that number words denote precise cardinalities rather than approximate ones. Already before they are able to count, young children realize that if you add items to a collection of "five", the resulting cardinality cannot be "five" anymore, and that "six plus more" is no longer "six", but that "a lot plus more" is still "a lot" (Sarnecka and Gelman 2004). As children grow older and have more experience with formal mathematics, they learn additional properties of numbers, such as that 2 is prime, or that 3 is both prime and odd. Knowledge-how involves elements of the counting routine, such as being able to recite number words from memory in the correct order. It also involves the ability to keep track of what is already counted when counting a collection of items. In many cultures, numerical competence includes being able to use correct procedures, for instance, to make calculations on an abacus or counting board, being able to carry numerals when multiplying, or using the correct procedure for a long division. Such skills require extensive practice, often in a formal school context.

\section{The child as a lone mathematician}

How do we explain young children's acquisition of knowledge about numbers, in particular the acquisition of CP? Standard accounts tend to conceptualize young children as lone mathematicians, who come up with the requisite concepts independently. This is in line with the popular view of the child as a little scientist, introduced by the developmental psychologist Piaget (1929), but going back as early as Rousseau (1762/1999). Piaget was impressed by how quickly children pick up information about their environment, and he was intrigued by the errors they made in the process. These errors suggested to him that children are not passive 
receptacles of knowledge, but active learners. Children learn by actively engaging with the world, and changing their ideas about it as a result of their experience, a bit like scientists who conduct experiments and formulate hypotheses on the basis of them. This view has contemporary defenders, for example, Gopnik, Meltzoff and Kuhl (2001), who draw an explicit comparison between babies and scientists engaged in individual experimentation. Carey $(2009,20)$ developes the analogy between scientists and children further, conceptualizing developmental changes in individual children's minds in terms of mini-scientific revolutions, with processes in individual minds being similar to "those described in the literature on history and philosophy of science" (Carey 2009, 20).

Carey $(2004,2009)$ regards children's acquisition of the CP as a cognitive revolution. She uses a Quinean bootstrapping account to explicate it. Bootstrapping is the process whereby a child acquires a new concept by first using a system of external symbols (counting words), initially without knowing what they mean. Through analogical and inductive inference, children come to realize what the symbols stand for. First, children learn the counting list as meaningless lexical items. During the next phase, they realize that "one" corresponds to one item in a collection (e.g., one biscuit), and they do the same with "two" and "three". Children probably rely on their subitizing capacity to associate the number words "one", "two", "three" (and sometimes "four") with the corresponding quantities. However, subitizing ends at 3 or 4, so for larger quantities this system does not work anymore, which may explain why children become one-knowers, two-knowers, three-knowers, and occasionally four-knowers, but never five-knowers. According to the bootstrapping account, it is at this point that a crucial induction takes place: the child notices an analogy between the next in the numeral list and next in series of objects to be counted. She realizes (for small numerosities within the subitizing range) if " $x$ " is followed by " $y$ " in the counting sequence, adding an individual to a set with cardinal value $x$ results in a set with cardinal value $y$. She generalizes this for quantities $>4$. In this way, the successor function is established. The successor function plays a crucial role in the Dedekind-Peano axioms of arithmetic, a $19^{\text {th }}$ century formalization of arithmetic. The successor function is a primitive function, which takes that if a given $n$ is a natural number, so is its successor, i.e., $S(1)=2, S(2)=3$, and so on. The successor function obeys several of the Dedekind-Peano axioms of arithmetic, for instance, that the successor of a natural number is also a natural number, and that there is no natural number whose successor is 0 . It is remarkable, given that this function was spelled out only in the $19^{\text {th }}$ century, that three- and four-year-old children around the world routinely hit upon this principle through induction.

The bootstrapping account is not without controversy. Rips et al. (2006), for example, have argued that it is insufficient to explain how children actually learn the natural numbers. They present a rather contrived case of three-year-old twins (Fran and Jan) to argue their case. Fran learns the standard natural numbers, while Jan learns a peculiar, modular system in which the count sequence cycles back around from "nine" to "none", and then goes back to "one", "two", etc., again. Clearly, Jan ends up with a badly underdetermined system, since "two" can refer to collections of 2 , $12,22, \ldots$ items. Although both children have used induction, only Fran learns the natural numbers. Thus, according to Rips et al. (2006), induction by itself does not 
suffice to establish natural numbers. Rips et al. (2008) instead propose that children use abstract mathematical schemas, such as commutativity $(a+b=b+a)$, to understand natural numbers. How do children learn such abstract schemas? According to Rips et al., they do so through physical experiences and manipulating the environment:

Suppose that children initially notice that two similar sets of objects for example, two sets of three toy cars - can be matched one-to-one. At a later stage, they may extend this matching to successively less similar objects - three toy cars matched to three toy drivers - and eventually to one-to-one matching for any two sets of three items. This could yield the general concept of sets that can be matched one-to-one to a target set of three objects - a possible representation for three itself (Rips 2008, 637).

Again, children are conceptualized as individual discoverers who actively experiment and acquire sophisticated mathematical knowledge through this.

The picture of preschoolers who learn about numbers, including sophisticated concepts as the successor function and commutativity is that of the lone mathematician-akin to Srinivasa Ramanujan (1887-1920) who, seemingly on his own, made substantial contributions to several areas of mathematics. The tradition of the child as lone scientist/mathematician is not only descriptive, but also normative: children ought to learn in this way. Educators, such as Maria Montessori, have devised education systems that encourage children to learn through individual discovery. The idea behind this is that individual discovery is a form of learning that is superior to social learning, which is regarded as mere parroting, that precludes a genuine understanding of what is being learned.

\section{Learning through testimony}

An alternative developmental picture sees young children primarily as social learners, who benefit from the vast knowledge and experience of adults and older children. This tradition goes back to Thomas Reid (1764) who regarded testimony as one of the most important channels through which we learn; like perception or memory, testimony is a basic source of knowledge. Although we may occasionally be led astray by liars or people who are genuinely mistaken, testimony is an important source of information for facts outside of our immediate experience. Indeed, without testimony, we would not even know the place and date of our own birth. Reid (1764) formulated several arguments for why testimony is a crucial and basic source of knowledge: humans have a natural inclination to believe what they learn from others, and they only become sceptical when they have reasons to be doubtful (e.g., the testifier is a known liar). Young children are more gullible than adults, showing that it is an innate tendency. As he wrote:

It is evident, that in the matter of testimony, the balance of human judgment is by nature inclined to the side of belief; and turns to that side of itself when there is nothing put in the opposite scale. ... if credulity were the effect of reasoning and experience, it must grow up 
and gather strength, in the same proportion as reason and experience do. But if it is the gift of nature, it will be strongest in childhood, and limited and restrained by experience; and the most superficial view of human life shows, that the last is really the case, and not the first (Reid 1764, 478-479).

Reid's account of testimony is both descriptive and normative: humans acquire vast stores of knowledge through testimony, and it is proper to do so. For testimony to work, two elements need to be in place: trustfulness and truthfulness, or as Reid calls them, the principle of credulity and the principle of veracity, "two principles that tally with each other" (Reid 1764, 474). Children need to be willing to learn counterintuitive, strange ideas, such as that the Earth is round even though it is in their own experience flat, if they want to learn the correct scientific view of the Earth. Parents and teachers must be "benevolent communicators" (Csibra and Gergely 2009, 152), who are willing to teach and not to deceive. The strange scenario Rips et al. (2006) offer of one of the twins being taught a modular counting method (by a "diabolic parent", as they put it) is unlikely to occur in real life.

This Reidian picture of children as trustful social learners is gaining increasing popularity in cognitive science (see e.g., Harris 2012, for a comprehensive overview). Csibra and Gergely (2009) have argued that humans are natural pedagogues, and that communication has evolved to facilitate the transmission of knowledge. Applying this model of the child as social learner to numerical cognition, we come to the view of children as socially-embedded mathematicians. Likening children to grown-up scientists or mathematicians is inherently problematic; for one thing, children do not really conduct experiments. However, even if one were to insist upon such a close analogy, it is important to point out that mathematicians rely on testimony and other forms of socially transmitted information. Thurston (2006) observes

Mathematical knowledge can be transmitted amazingly fast within a subfield. When a significant theorem is proved, it often (but not always) happens that the solution can be communicated in a matter of minutes from one person to another within the subfield. The same proof would be communicated and generally understood in an hour talk to members of the subfield ... When people are doing mathematics, the flow of ideas and the social standard of validity is much more reliable than formal documents. People are usually not very good in checking formal correctness of proofs, but they are quite good at detecting potential weaknesses or flaws in proofs (Thurston, $2006,43,46)$.

Geist et al. (2010) argue that mathematicians frequently rely on testimony to decide matters such as the soundness of a mathematical proof. For instance, many proofs rely on theorems that have been proved before, and individual mathematicians using these theorems do not meticulously check for themselves whether these earlier proofs are correct. Moreover, reviewers for papers for mathematical journals 
do not check all proofs in detail for themselves, but rely on the author's own scrutiny, only checking results in detail when it seems warranted to do so.

Given that children's early knowledge of numerical concepts is largely procedural (correctly using the counting routine to denote cardinalities of sets), one may ask whether testimony is a good model for the acquisition of number concepts. When we think of testimony, we tend to think of propositional knowledge. Indeed, Poston (2016) argues that knowing-how is not transmitted as easily as knowing-that. When I tell my daughter that Jimi Hendrix was a famous guitarist, she thereby comes to know that Hendrix was a famous guitarist. However, when someone demonstrates a guitar riff from Hendrix' repertoire to her, she thereby does not come to know that riff. It would likely take her many weeks to learn the riff, and if her guitar skills are not up to it, she may never learn how to perform it correctly. We can through testimony learn fairly complex pieces of propositional knowledge, which does not seem to be the case for knowledge-how. This disanalogy between the transmission of knowing-that (which you can do by simply stating that $p$ ) and knowing-how (which you cannot do by simply demonstrating $\phi$ ) leads Poston (2016) to conclude that knowing-how is not reducible to knowing-that.

While Poston (2016) is correct that learning a skill requires additional resources from the learner (e.g., practice), there are nonetheless striking parallels between verbal testimony and demonstration of knowing-how. Buckwalter and Turri (2014) argue that demonstration has a close analogue to the knowledge norm of assertion. According to the knowledge norm of assertion, when you assert that $p$ you should know that $p$ (Williamson 2000). There are several motivations for this, e.g., it would be paradoxical to say: $p$, but I don't know that $p$, and people will often challenge an assertor by asking, "How do you know that?" Similarly, in showing a skill (demonstration), you need to know how to perform that skill. It would be paradoxical to say, "I will show you how to $\phi$, but I don't know how to $\phi$ ". You can also challenge a demonstrator, e.g., one can ask a bungling ski instructor "Do you actually know how to ski?"

If we look at testimony in the broader context of social learning, it is clear that demonstrating skills preceded verbal testimony, perhaps by over one million years. Evidence for explicit teaching and demonstration appears as early as 2.5 million years ago. Early human stone tool technology, such as Oldowan (ca. 2.5 million years ago) and Acheulean (ca. 1.5 million years ago), require some form of social learning that almost certainly involved demonstration, and very likely also explicit teaching, e.g., how to correctly strike the core (demonstrating the angle with which you have to hit the core so as to obtain a razor-sharp flake that can be used to cut meat), or which pebbles to flake from. Demonstration and trusting demonstration may be a uniquely human capacity. While chimpanzees are sophisticated social learners, they do not faithfully follow demonstrators. For example, Horner and Whiten (2005) showed children and juvenile chimpanzees a complex way to open a puzzle box in order to retrieve a candy. Both chimps and children opened the box in the way that was demonstrated. However, in a second try, a transparent box was used that made it obvious that part of the actions were superfluous. The children continued using the way that was demonstrated to them (this tendency of sticking to a learned 
suboptimal routine is called overimitation). The chimpanzees went for a more direct way to obtain the candy that deviated from the demonstration. The human default position is one of trust. Children show a high degree of trust when an adult demonstrates them the "proper way" to do something: they assume, by default, that demonstration presupposes knowing-how. Overimitation has not only been found in western children (who might be influenced by schooling), but also in children who live in groups with little formal education, such as in South-African and Australian hunter-gatherer societies (Nielsen et al. 2014).

Humans are the only species that have cumulative cultural transmission in a variety of domains: they build on the accomplishments of earlier generations and create more complex cultural elements, including artifacts (e.g., the atl atl, a small artifact that attaches to a spear to make it go much further and faster when thrown), ways of preparing food (to make it less toxic, tastier, or easier to digest), and stories (including very long and complex epic tales). Cumulative culture is not altogether absent in nonhuman animals. For example, New Caledonian crows (Corvus moneduloides) which use tools made of the edges of Pandanus leaves to help them catch invertebrates, use design innovations by conspecifics to improve their own tools (Hunt and Gray 2003). However, their cumulative culture is limited to the domain of tools. By contrast, human learning takes place in an environment populated with potential teachers and artifacts, and concerns a wide range of domains. According to Sterelny's (2012) apprenticeship model, humans grow up in environments that are "seeded" with artifacts that help and support learning novel skills and information. Children learn by combining information from the social world with information from the physical world. For example, in a workshop, tools tend to be laid out in an order that facilitates completing specific tasks, such as making or repairing furniture. Through observing accomplished woodworkers or through active teaching, or likely a combination of both, young apprentices learn both the requisite propositional knowledge (e.g., distinguishing between the top rail, splat, and back rail of a chair) and knowledge-how (e.g., how to use a hole-saw properly). In this way, the testimonial transmission of many complex domains of culture requires both a transmission of propositional knowledge, and a transmission of skills through demonstration.

\section{The testimonial transmission of number concepts}

We are now in a position to examine how the testimonial transmission of number concepts might work. When children learn to count at about 3.5-4 years of age, they do not yet have a fully-fledged concept of natural numbers. For example, children of this age tend to fail the Piagetian conservation task (Piaget 1941): when one lays out a row of items, they tend to think that its number changes if the spatial configuration of the row changes (e.g., by widening the gaps between the items). Although Piaget's theoretical rationale for why young children fail this task, and only succeed in it about age 5-7 is no longer generally accepted, failure of toddlers to pass the conservation task is robust and has often been replicated. Young CPknowers do not realize that number words further in the counting list are larger than numbers earlier in the counting list (the later-greater principle), even though it 
seems like this could be easily inferred from the CP and its applications (Le Corre 2014).

Moving to more sophisticated concepts, the development of children's concept of zero follows a different trajectory compared to other natural numbers. It seems to proceed in three phases (Wellman and Miller 1986). In the earliest phase, preschoolers recognize the Arabic numeral 0 and the noun "zero", but they do not know what it means. In the second phase, children understand zero to refer to nothing, but they do not understand its relationship to other small numbers (e.g., they are equally likely to say that 0 is smaller than 3 or vice versa, whereas they understand that 1 is smaller than 3). The third phase is when children realize zero is the smallest natural number, and they can make accurate comparisons between zero and other small numbers.

Children's understanding of infinity also appears several years after they learn to count. Many counting systems, be they number words or other ways to denote numbers (e.g., pointing to body parts, where each body part symbolizes a natural number) are quite limited, often not exceeding 20 or 30 . In these cultures, people can come up with higher numbers on an ad hoc basis (e.g., counting fingers and toes of several people present). Children's understanding of infinity only arises at about 8 years of age, many years after their ability to count collections has developed. Eightyear-olds have some understanding of potential and actual infinity, but they still do not appreciate that an infinite set is immeasurably bigger than a finite set (for example, they mistakenly think that a very large set, such as the number of grains of sand on a beach, is almost infinite)-indeed, even many numerate adults fail to grasp this unbridgeable gap between very large sets and infinite sets (Falk 2010). It is worth pointing out that the concepts of zero and infinity are lacking in many cultures that use natural numbers (e.g., Roman numerals do not have a symbol for zero). Thus, knowledge of numbers is not all-or-nothing. It has many components, such as understanding how counting works, what cardinalities are, how to perform calculations, as well as more complex, culturally restricted concepts, such as zero and infinity.

How do children learn about natural numbers in a social context? Children tend to grow up in environments that are seeded with artifacts and ideas that help them acquire number concepts. These include not only special learning tools, such as abacuses for children, or foam numbers for the bathtub, but also the symbolic representations of numbers that adults use in their day-to-day lives. In this environment, children acquire knowledge of numbers through their parents, older siblings, and more formal learning situations (e.g., preschool and elementary school).

Learning the counting list by rote memory is a crucial step towards learning about natural numbers. Counting is ostensive and deliberate: across the world, there are counting songs and routines to help children memorize the first few counting words. In English, this is usually the first five (Five little monkeys, Five little ducks) or the first ten counting words (Ten in a bed, This old man). During these early encounters, children do not yet grasp the semantic content of these number words, but the songs do help them familiarize themselves with counting routines. Interestingly, 
such counting routines and songs are absent in languages without exact number words, and in homesigners (deaf children and adults who live with hearing parents and carers, without using a fully-developed sign language) (Spaepen et al. 2011).

Levine et al. (2010) recorded 7.5 hours of natural conversations (in several sessions) between parents and toddlers aged 14 to 30 months to understand how talking about numbers ("number talk") influences numerical cognition. Parents were informed that this was a study on language development, but they did not learn that it was specifically about number words, so as to not influence them to use more number words than they would do under ordinary circumstances. The authors found that parents used an average of 90.8 number words over the 7.5 hours recorded. Remarkably, only an average of 6.3 instances of this number talk consisted of prompts where children had to give cardinal values, e.g., "how many cars do you see?" Most of the time, number talk was testimonial. About $50 \%$ of number talk by parents consisted of stating cardinal values ("Look, three fish!"); $32 \%$ consisted of counting songs, rhymes and routines. When the toddlers engaged in number talk, about $62 \%$ consisted of counting routines, and $28 \%$ of cardinal values. These utterances demonstrate the importance of transmitting knowing-how to count in children's early acquisition of number concepts. Both the toddlers and their parents included counting routines in their number talk. Levine et al. (2010) found that children whose parents engaged more in number talk performed better at a cardinality test at 46 months. Given that high socioeconomic status (SES) parents tend to talk more to their children, they controlled for the amount of talking and for SES. They found that parents who talked more about number, taking into account SES, had children with more knowledge of the cardinal meanings of number words at 46 months.

Number talk not only helps children to master the counting routine, it also helps them to become aware of the fact that number words and symbols refer to discrete quantities. This has been demonstrated in the blocks and water task (Slusser et al. 2013), where young children are presented with two bowls, one containing countable items (blocks of different colors) and another containing colored water. The experimenter asks the toddlers either a number question "Which one has five?" or a question that does not involve numbers ("Which one has orange?") N-knowers, who can only enumerate collections up to three or four, do well within their range but perform less consistently at higher numbers (five, six). However, when experimenters start with small numbers and work their way up, these younger children tend to choose the bowl with the blocks when asked "Which one has five/six", indicating they have some understanding that these cardinalities refer to discrete entities, not to uncountable quantities. Number talk, where parents, other carers, and older siblings refer to discrete sets, may help young children to understand this property of number words.

Next to linguistic input, artifacts can help support children's developing numerical cognition. Board games, such as the Game of Goose, which often involve numerical properties (e.g., throwing a die that shows $n$ requires the child to move her piece $n$ spaces forward), have a positive impact on children's later mathematical achievements. As Siegler and Booth $(2004,441)$ point out, this may be because 
"Board games provide children with strongly correlated spatial, temporal, kinaesthetic, and verbal or auditory cues to numerical magnitude." Siegler and Ramani (2009) found that especially designed games that were linear, rather than having numbers in a spiraling or circular pattern, were particularly effective in helping young children from low SES backgrounds to close the numeracy gap with their high SES peers. Under the apprenticeship model of human learning, the environment is seeded with artifacts that help to support the development of numeracy. However, the lack of knowledge of natural numbers in homesigners indicates that an environment seeded with cues for numbers is not sufficient. There needs to be active testimonial transmission as well, which is lacking in these transmission situations where there is no adequate sign language to transmit number concepts.

If the acquisition of number concepts is the result of testimony to skills and propositional knowledge, we can understand why not all human cultures have natural numbers. Some cultures, such as the Pirahã (e.g., Frank et al. 2008) and the Mundurucú (e.g., Pica et al. 2004), lack words to denote exact cardinalities. In a video ${ }^{1}$ recorded by anthropologist Pierre Pica, an old man and the medicine man of a Mundurucú village attempt to count 10 and 13 seeds, with limited success. As can be seen, the men are not used to count, e.g., they do not separate what has already been counted and what still has to be counted, something young children in numerate cultures learn early on. Mundurucú do not have counting routines, although, intriguingly, their language does have a count/mass noun distinction, indicating they see the difference between discrete and continuous quantities (Pica and Lecomte 2008). While this distinction is vital for children to understand natural numbers, it is clearly not enough to help them count.

The testimonial model of knowledge transmission can also explain why non-human animals fail to understand $\mathrm{CP}$. Learning $\mathrm{CP}$, as we have seen, depends on an understanding of the counting routine. Children learn the counting routine when they are young toddlers, far too early to grasp its meaning. Humans are willing to copy actions even if they do not understand them, which helps them to acquire the correct sequence of counting words. This is a big difference with the chimpanzee Ai who was taught to use Arabic digits in Biro and Matsuzawa's (2001) studies. Ai did not learn the counting routine, instead relying on estimation to enumerate collections of items and assigning them to the correct numeral. As Biro and Matsuzawa (2001) observe, while Ai may have been similar to children in the fact that she learned symbols for numbers, this is just one aspect of learning how to count. She was not raised in a supportive, rich environment filled with numerical cues.

\section{Conclusions}

The question of how children learn number concepts is unresolved. In this paper, I have argued that testimony plays an important role in the transmission of number concepts', both to knowing-how (the skills involved in counting) and to knowing-that

\footnotetext{
${ }^{1}$ https://www.youtube.com/watch?v=9iXh8wte3gM
} 
(propositional knowledge about number words, such as that they refer to discrete quantities). Children grow up in a world seeded with artifacts, counting songs, and other cultural features that help them to count. I have pointed out the shortcomings of the model of children as lone mathematicians, who discover sophisticated mathematical principles by themselves. In order to understand numerical cognition, we should not only pay attention to what may go on in the minds of young children, but also to what goes on in their broader learning environment.

Acknowledgments

Many thanks to Sorin Bangu, Max Jones, Dirk Schlimm, Johan De Smedt, and audiences in Bergen, Norway and Bristol, for their helpful comments to an earlier version of this paper.

\section{References}

Antell, S.E., \& Keating, D.P. (1983). Perception of numerical invariance in neonates. Child Development, 54, 695-701.

Biro, D., \& Matsuzawa, T. (2001). Chimpanzee numerical competence: Cardinal and ordinal skills. In T. Matsuzawa (Ed.), Primate origins of human cognition and behavior (pp. 199-225). Tokyo: Springer.

Bisazza, A., Piffer, L., Serena, G., \& Agrillo, C. (2010). Ontogeny of numerical abilities in fish. PLOS ONE, 5, e15516.

Buckwalter, W., \& Turri, J. (2014). Telling, showing and knowing: A unified theory of pedagogical norms. Analysis, 74, 16-20.

Cain, C. R. (2006). Implications of the marked artifacts of the Middle Stone Age of Africa. Current Anthropology, 47, 675-681.

Carey, S. (2004). Bootstrapping and the origin of concepts. Daedalus, 133, 59-68.

Carey, S. (2009). The origin of concepts. Oxford: Oxford University Press.

Csibra, G., \& Gergely, G. (2009). Natural pedagogy. Trends in Cognitive Sciences, 13, 148-153.

Dacke, M., \& Srinivasan, M.V. (2008). Evidence for counting in insects. Animal Cognition, 11, 683-689.

Davidson, K., Eng, K., \& Barner, D. (2012). Does learning to count involve a semantic induction? Cognition, 123, 162-173.

Falk, R. (2010). The infinite challenge: Levels of conceiving the endlessness of numbers. Cognition and Instruction, 28, 1-38.

Frank, M. C., Everett, D. L., Fedorenko, E., \& Gibson, E. (2008). Number as a cognitive technology: Evidence from Pirahã language and cognition. Cognition, 108, 819-824. 
Geist, C., Löwe, B., \& Van Kerkhove, B. (2010). Peer review and knowledge by testimony in mathematics. In B. Löwe, \& T. Müller (Eds.), PhiMSAMP. Philosophy of mathematics: Sociological aspects and mathematical practice (pp. 155-178). London: College Publications.

Gopnik, A., Meltzoff, A. N., \& Kuhl, P. (2001). The scientist in the crib. What early learning tells us about the mind. New York: Perennial.

Harris, P.L. (2012). Trusting what you're told. How children learn from others. Cambridge, MA: Harvard University Press.

Horner, V., \& Whiten, A. (2005). Causal knowledge and imitation/emulation switching in chimpanzees (Pan troglodytes) and children (Homo sapiens). Animal Cognition, 8, 164-181.

Hunt, G. R., \& Gray, R. D. (2003). Diversification and cumulative evolution in New Caledonian crow tool manufacture. Proceedings of the Royal Society of London B: Biological Sciences, 270, 867-874.

Le Corre, M. (2014). Children acquire the later-greater principle after the cardinal principle. British Journal of Developmental Psychology, 32, 163-177.

Levine, S.C., Suriyakham, L.W., Rowe, M.L., Huttenlocher, J., \& Gunderson, E.A. (2010). What counts in the development of young children's number knowledge? Developmental Psychology, 46, 1309-1319.

Nielsen, M., Mushin, I., Tomaselli, K., \& Whiten, A. (2014). Where culture takes hold: "Overimitation" and its flexible deployment in Western, Aboriginal, and Bushmen children. Child Development, 85, 2169-2184.

Petrazzini, M. E. M., Lucon-Xiccato, T., Agrillo, C., \& Bisazza, A. (2015). Use of ordinal information by fish. Scientific Reports, 5, 15497.

Piaget, J. (1929). The child's conception of the world (J. Tomlinson and A. Tomlinson, trans). London: Routledge and Kegan Paul.

Piaget, J. (1941/1952). The child's conception of number (trans. C. Gattegno and F.M. Hodgson). London and New York: Routledge.

Pica, P., \& Lecomte, A. (2008). Theoretical implications of the study of numbers and numerals in Mundurucu. Philosophical Psychology, 21, 507-522.

Pica, P., Lemer, C., Izard, V., \& Dehaene, S. (2004). Exact and approximate arithmetic in an Amazonian indigene group. Science, 306, 499-503. 
Pletser, V., \& Huylebrouck, D. (1999). The Ishango artefact: The missing base 12 link. Forma, 14, 339-346.

Poston, T. (2016). Know how to transmit knowledge? Noûs, 50, 865-878.

Reid, T. (1764). An Inquiry into the Human Mind, on the Principles of Common Sense. Edinburgh: Millar, Kincaid \& Bell.

Revkin, S.K., Piazza, M., Izard, V., Cohen, L., \& Dehaene, S. (2008). Does subitizing reflect numerical estimation? Psychological Science, 19, 607-614.

Rips, L.J., Asmuth, J., \& Bloomfield, A. (2006). Giving the boot to the bootstrap: How not to learn the natural numbers. Cognition, 101, B51-B60.

Rips, L.J., Bloomfield, A., \& Asmuth, J. (2008). From numerical concepts to concepts of number. Behavioral and Brain Sciences, 31, 623-642.

Rousseau, J-J. (1762/1999). Emile. In Oeuvres Complètes, Volume 4. Paris: Pléiade.

Rugani, R., Vallortigara, G., \& Regolin, L. (2013). Numerical abstraction in young domestic chicks (Gallus gallus). PloS One, 8, e65262.

Sarnecka, B.W., \& Gelman, S.A. (2004). Six does not just mean a lot: Preschoolers see number words as specific. Cognition, 92, 329-352.

Sarnecka, B.W. (in press). Learning to represent exact numbers. Synthese.

Scarf, D., Hayne, H., \& Colombo, M. (2011). Pigeons on par with primates in numerical competence. Science, 334, 1664-1664.

Siegler, R.S., \& Booth, J.L. (2004). Development of numerical estimation in young children. Child Development, 75, 428-444.

Siegler, R.S., \& Ramani, G. B. (2009). Playing linear number board games-but not circular ones-improves low-income preschoolers' numerical understanding. Journal of Educational Psychology, 101, 545-560.

Slusser, E., Ditta, A., \& Sarnecka, B. (2013). Connecting numbers to discrete quantification: A step in the child's construction of integer concepts. Cognition, 129, 31-41.

Spaepen, E., Coppola, M., Spelke, E. S., Carey, S. E., \& Goldin-Meadow, S. (2011). Number without a language model. Proceedings of the National Academy of Sciences, 108, 3163-3168.

Sterelny, K. (2012). The evolved apprentice. How evolution made humans unique. Cambridge, MA: MIT Presss.

Thurston, W. (2006). On proof and progress in mathematics. In R. Hersh (Ed.), 18 unconventional essays on the nature of mathematics (pp. 37-55). New York: Springer. 
Tomasello, M., Carpenter, M., Call, J., Behne, T., \& Moll, H. (2005). Understanding and sharing intentions: The origins of cultural cognition. Behavioral and Brain Sciences, 28, 675-691.

Williamson, T. (2000). Knowledge and its limits. Oxford: Oxford University Press.

Wellman, H.M., \& Miller, K.F. (1986). Thinking about nothing: Development of concepts of zero. British Journal of Developmental Psychology, 4, 31-42.

Wynn, K. (1990). Children's understanding of counting. Cognition, 36, 155-193. 